\title{
Assessing a Tornado Climatology from Global Tornado Intensity Distributions
}

\author{
BERNOLD FEUERSTEIN \\ Max-Planck-Institut für Kernphysik, Heidelberg, Germany \\ NiKolai DotZeK \\ Institut für Physik der Atmosphäre, DLR, Oberpfaffenhofen, Wessling, Germany \\ JÜRGEN GRIESER \\ Deutscher Wetterdienst, Offenbach, Germany
}

(Manuscript received 9 February 2004, in final form 29 July 2004)

\begin{abstract}
Recent work demonstrated that the shape of tornado intensity distributions from various regions worldwide is well described by Weibull functions. This statistical modeling revealed a strong correlation between the fit parameters $c$ for shape and $b$ for scale regardless of the data source. In the present work it is shown that the quality of the Weibull fits is optimized if only tornado reports of F1 and higher intensity are used and that the $c-b$ correlation does indeed reflect a universal feature of the observed tornado intensity distributions. For regions with likely supercell tornado dominance, this feature is the number ratio of F4 to $\mathrm{F} 3$ tornado reports $R(\mathrm{~F} 4 / \mathrm{F} 3)=0.238$. The $c-b$ diagram for the Weibull shape and scale parameters is used as a climatological chart, which allows different types of tornado climatology to be distinguished, presumably arising from supercell versus nonsupercell tornadogenesis. Assuming temporal invariance of the climatology and using a detection efficiency function for tornado observations, a stationary climatological probability distribution from large tornado records (U.S. decadal data 1950-99) is extracted. This can be used for risk assessment, comparative studies on tornado intensity distributions worldwide, and estimates of the degree of underreporting for areas with poor databases. For the 1990s U.S. data, a likely tornado underreporting of the weak events (F0, F1) by a factor of 2 can be diagnosed, as well as asymptotic climatological $c, b$ values of $c=1.79$ and $b=2.13$, to which a convergence in the 1950-99 U.S. decadal data is verified.
\end{abstract}

\section{Introduction}

Tornadic storms are a prominent form of severe weather, causing significant to devastating damage to man-made structures, forests, etc. They occur in all regions worldwide where thunderstorms are known. Thus, knowledge about tornado intensity distributions is important for both basic climatology research and practical issues like risk assessment (Brooks and Doswell 2001a) and the insurance industry (Dotzek 2002). Intensity of tornadoes is measured either by the Fujita scale (F scale; Fujita and Pearson 1973; Fujita 1981) or the twice-as-fine Tornado and Storm Research Organisation (TORRO) scale (T scale; Meaden 1976). The F scale, which will be used throughout this paper,

Corresponding author address: Dr. Bernold Feuerstein MaxPlanck-Institut für Kernphysik, Saupfercheckweg 1, 69117 Heidelberg, Germany.

E-mail: feuerstb@mpi-hd.mpg.de distinguishes two intensity classes at a time for weak (F0, F1), strong (F2, F3), and violent (F4, F5) tornadoes (Kelly et al. 1978). In the original definition by Fujita and Pearson (1973) and the review by Fujita (1981), the Fujita $(F)$ scale is defined formally as equal to the Beaufort $^{1}(B)$ scale as $\left(v\right.$ in $\left.\mathrm{m} \mathrm{s}^{-1}\right)$

$$
v(B)=0.84 B^{3 / 2}, v(F)=6.30(F+2)^{3 / 2} .
$$

It defines $F=1$ as the low end of hurricane force winds $\left(33 \mathrm{~m} \mathrm{~s}^{-1}\right)$ and $F=12$ as mach $1\left(330 \mathrm{~m} \mathrm{~s}^{-1}\right)$. In practice, the useful range goes from $F=0$ to $F=6$. Note that the Fujita scale classes $(\mathrm{F} 0, \mathrm{~F} 1, \ldots, \mathrm{F} 5)$ are discrete, while the italicized $F$ denotes a continuous

\footnotetext{
${ }^{1}$ The original description of the Beaufort scale considers the effect of a 10-min-averaged wind speed over sea, while the Fujita and TORRO scales are related to the peak wind speeds. However, the velocity-based definition of the Beaufort scale in Eq. (1) is applied to both average and peak wind speeds as, for example, for weather forecasts.
} 
variable. So with the wind speed-based definition given above, $F=-2$ corresponds to $v=0$, the $\mathrm{F}$ classes comprise integer intervals in $F$ (e.g., F0 ranges $0 \leq F<$ 1 , and the central value of class $\mathrm{F}_{n}$ is $F=n+0.5$.

However, due to a lack of direct wind speed information, in practice the F-scale rating is almost exclusively based on observed maximum damage at a given point. This issue has been, and is still, under discussion (Doswell and Burgess 1988; Brooks and Doswell 2001b; McDonald 2002), and preliminary concepts to include information on the strength of man-made structures (Fujita 1992; Dotzek et al. 2000; Dotzek 2001) as well as tree damage (Hubrig 2002; Hubrig 2004) have been developed.

Recent studies revealed that tornado intensity distributions for the United States and many countries in Europe, South America, and other regions worldwide show a similar, quasi-exponential behavior (Brooks 2000; Brooks and Doswell 2001b; Dotzek 2001; Dotzek 2002). Nevertheless, it remained unclear whether an exponential distribution is indeed an appropriate description for tornado intensities. In their previous work, Dotzek et al. (2003) applied a new statistical modeling in a comprehensive manner to the observed intensity distributions from various regions worldwide. It turned out that present tornado intensity distributions seem not to be described properly by exponentials, as they show curvature to the right in lin-log plots, even for large databases. Furthermore, exponentials do not fulfill the physical boundary condition of zero tornadoes with zero wind speed and cannot reflect the presence of an upper limit to tornado intensities near the F5-F6 threshold following from energy budget calculations. Both can be satisfied by Weibull distributions, which still encompass exponentials as a special case. A Weibull distribution, which is often used with extreme values, "ordinary" wind speeds, and even for distributions of tornado pathlength and width (Brooks 2004), is given in the following three-parameter form for the probability density $p(x)$ and probability:

$$
\begin{aligned}
& P(x)=\int_{a}^{x} p\left(x^{\prime}\right) d x^{\prime} \\
& p(x)=\frac{c}{b}\left(\frac{x-a}{b}\right)^{c-1} \exp \left[-\left(\frac{x-a}{b}\right)^{c}\right], \quad \forall x>a \\
& P(x)=1-\exp \left[-\left(\frac{x-a}{b}\right)^{c}\right], \quad \forall x>a
\end{aligned}
$$

Here $a$ denotes the lower boundary in the variable $x$ (either $v$ or $F$ ); $b$ is a scaling factor, and $c$ a shape parameter. For $c=1$, Eq. (2a) reduces to an exponential distribution. Physical and statistical considerations suggest the inclusion of negative F-scale values in the intensity analysis, that is, to apply the scales down to $v$ $=0 \mathrm{~m} \mathrm{~s}^{-1}$ as originally proposed by Fujita and Pearson
(1973). Using Fujita's $v(F)$ relation from Eq. (1), this leads to $a=-2$ for a Weibull distribution in $F$. Dotzek et al. (2003) demonstrated that Weibull fits in either $v$ or $F$ reproduce the observations significantly better than exponentials. For each region and time frame under investigation the estimated parameters $b$ and $c$ of the Weibull fit were plotted in a $c-b$ diagram. Therefore each data source is represented by a corresponding point. Structures within this diagram provide information about tornado climatology, for example, a random distribution of $c, b$ points would indicate that there is neither a temporal nor a spatial relation between different databases. The occurrence of clusters would reveal different groups with similar properties of the tornado intensity distributions. However, Dotzek et al. (2003) have found a strong correlation between the $c$ and $b$ values, indicating a "universal" climatological property. For the large (>70 yr) U.S. database, the temporal evolution of the $c$ and $b$ values was investigated over several decades. This revealed a convergence toward an asymptotic climatological intensity distribution.

The latter issues were the main motivation for the present paper. In section 2 , we address the question of how the observed $c-b$ correlation is related to a climatological invariant. Using the large U.S. database and by introducing a detection efficiency function for tornadoes, a climatological Weibull intensity distribution for presumably supercell-dominated tornadogenesis is derived. Section 3 considers the problem of F0 underreporting and rating problems and how the fitting procedure can be optimized. In section 4 , the previously proposed characterization of intensity distributions via analyzing the average slope in lin-log plots is extended to an analysis of the neighboring F-class occurrence ratio. This suggests using the $c-b$ diagram for an identification of climatologically distinct areas (e.g., supercell versus nonsupercell tornadoes). Sections 5 and 6 present a discussion and conclusions.

\section{Correlation of the Weibull parameters $b$ and $c$ for tornado intensity distributions}

\section{a. An invariant property of the distributions}

Figure 1 shows a diagram for the Weibull parameters $c$ (shape) and $b$ (scale) derived from a fitting procedure to individual tornado intensity datasets from various regions worldwide (cf. Fig. 6 and Tables 5 and 6 from Dotzek et al. 2003). A more detailed discussion of the fitting procedure is given in section 3 of this work.

Part of the F-scale data for this analysis was extracted from Goliger et al. (1997), Peterson (2000), and Teittinen (2000). Updated numbers of the Japanese tornado climatology (cf. Niino et al. 1997), for Ireland and the United Kingdom, were kindly provided for this study within the European Severe Storms Laboratory (ESSL) network (information available online at http:// 


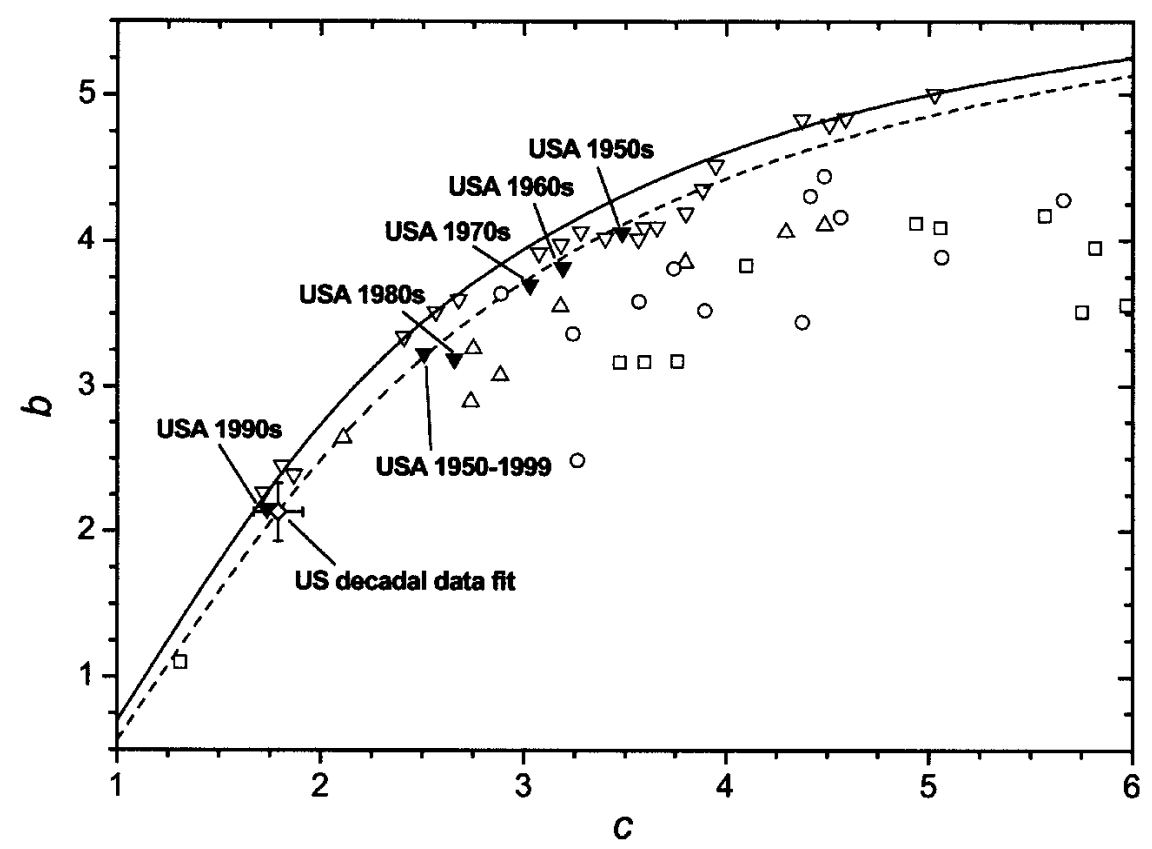

FIG. 1. A $c-b$ diagram of Weibull parameters $c$ and $b$ for fits in $F$ starting at $\mathrm{F}-2$ (i.e., $v=$ $0 \mathrm{~m} \mathrm{~s}^{-1}$, from Dotzek et al. 2003). Maximal observed tornado intensity: $\nabla, \mathrm{F} 5 ; \triangle, \mathrm{F} 4 ; \square, \mathrm{F} 3$; $\mathrm{O}$, databases with poor statistics. Curves of the constant F4/F3 occurrence ratio: solid line, $R$ $=0.238$ (from regions with F5); dashed line, $R=0.177$ (from U.S. decadal fit).

essl.org). The sources for the remaining countries were already given by Brooks and Doswell (2001b). For large databases similar to that from the United States splitting the data into individual decades was possible. For most non-U.S. countries having smaller databases, all data from the whole time period with rated tornadoes had to be included to yield a reasonable sample size. The data records, their time periods, and their sample sizes are given in Table 1.

The data vary both in the number of observed F-scale intensities and in the total number of tornadoes. Concerning the maximum reported $\mathrm{F}$-scale value for a given region and time frame we distinguish different classes of datasets as indicated by the symbols in Fig. 1 (observations extending to F3, F4, or F5 events, respectively, and small databases with poor statistics, i.e., less than a total of 100 events, excluding F0). The $c, b$ data points from regions with observed F5 events follow a curved line, whereas in particular those without F4 and F5 events lie significantly below this line. The points corresponding to statistically poor databases exhibit an irregular scattering and, thus, will be excluded from the following analysis.

The line of points formed by the F5-included databases suggests an interdependency of the parameters $c$ and $b$, which comes unexpected since they are, by definition of the Weibull distribution, independent parameters. Thus, this relation appears to reflect a general, climatologically relevant property of the corresponding intensity distributions. If so, such a property must be invariant-both regionally and temporally. One possible candidate is the occurrence ratio for neighboring F-scale classes $R\left(\mathrm{~F}_{n} / \mathrm{F}_{n-1}\right)=N(F) / N(F-1)$, where $N(F)$ denotes the number of reported events for an intensity class $F$. We choose the $R(\mathrm{~F} 4 / \mathrm{F} 3)$ ratio since it possesses a reasonable statistical sample but is, at the same time, not too strongly affected by the $F$-dependent detection probability. The latter refers to the fact that weak tornadoes are likely often overlooked since they are, on average, shorter lived and cause less significant damage (see next subsection for a more detailed discussion).

Considering all datasets from regions with documented F5 events, which are supposed to have a similar climatology and good statistical quality, we find $R(\mathrm{~F} 4 /$ $\mathrm{F} 3)=0.238(13) .^{2}$ Based on the Weibull probability density distribution from Eq. (2a), we approximate the tornado occurrence per F-scale class by

$$
\begin{aligned}
N(F)= & N_{0} p(F+2)=N_{0} \frac{c}{b}\left(\frac{F+2}{b}\right)^{c-1} \\
& \exp \left[-\left(\frac{F+2}{b}\right)^{c}\right] .
\end{aligned}
$$

\footnotetext{
${ }^{2}$ Throughout this paper, uncertainties (error bars) of numerical values are given in parentheses for the last significant decimals, for example, $0.238(13)=0.238 \pm 0.013$, or $13.8(20)=13.8 \pm 2.0$, respectively.
} 
TABLE 1. Weibull parameters $c$ and $b$ for fits in $F$ starting from $\mathrm{F}-2\left(v>0 \mathrm{~m} \mathrm{~s}^{-1}\right)$ using data from various regions worldwide and two fitting procedures (including and excluding F0 data, respectively). Sample size $N$, maximum observed F-scale intensity, and $\chi^{2}$ values are also shown. The last column gives a tentative assignment for the climatology being of type A, B, or C (see Fig. 6 and text).

\begin{tabular}{|c|c|c|c|c|c|c|c|c|c|}
\hline \multirow[b]{2}{*}{ Region, time } & \multirow[b]{2}{*}{$N$} & \multirow[b]{2}{*}{$F_{\max }$} & \multicolumn{3}{|c|}{$\begin{array}{l}\text { Weibull fit in } F \\
\text { (F0 included) }\end{array}$} & \multicolumn{3}{|c|}{$\begin{array}{l}\text { Weibull fit in } \\
F(\text { F0 excluded })\end{array}$} & \\
\hline & & & $c$ & $b$ & $\chi^{2}$ & $c$ & $b$ & $\chi^{2}$ & \\
\hline United States $1950-99$ & 39929 & 5 & 2.508 & 3.224 & 0.0027 & 2.416 & 3.124 & 0.0021 & $\overline{\mathrm{A}}$ \\
\hline United States $1950-82$ & 20993 & 5 & 3.239 & 3.854 & 0.0277 & 2.645 & 3.389 & 0.0034 & A \\
\hline United States 1990s & 12139 & 5 & 1.735 & 2.151 & 0.0027 & 1.927 & 2.467 & 0.0020 & A \\
\hline United States 1980s & 8192 & 5 & 2.658 & 3.185 & 0.0097 & 2.407 & 2.924 & 0.0048 & A \\
\hline United States 1970s & 8652 & 5 & 3.029 & 3.695 & 0.0245 & 2.412 & 3.136 & 0.0004 & A \\
\hline United States 1960s & 7031 & 5 & 3.191 & 3.814 & 0.0021 & 3.028 & 3.698 & 0.0003 & A \\
\hline United States 1950s & 4915 & 5 & 3.485 & 4.054 & 0.0168 & 3.064 & 3.787 & 0.0015 & A \\
\hline United States 1940s & 1649 & 5 & 4.373 & 4.831 & 0.0364 & 4.629 & 4.903 & 0.0348 & A \\
\hline United States 1930s & 1792 & 5 & 3.947 & 4.516 & 0.0289 & 4.024 & 4.548 & 0.0296 & A \\
\hline United States 1920s & 1391 & 5 & 5.025 & 5.005 & 0.1570 & 4.046 & 4.755 & 0.0348 & A \\
\hline Oklahoma 1950-99 & 2893 & 5 & 2.678 & 3.595 & 0.0029 & 2.742 & 3.652 & 0.0028 & A \\
\hline Oklahoma 1990s & 720 & 5 & 1.810 & 2.452 & 0.0250 & 2.605 & 3.504 & 0.0095 & A \\
\hline Oklahoma 1980s & 515 & 5 & 2.409 & 3.337 & 0.0052 & 2.208 & 3.100 & 0.0031 & A \\
\hline Oklahoma 1970s & 474 & 5 & 3.884 & 4.349 & 0.0466 & 3.314 & 4.062 & 0.0090 & A \\
\hline Oklahoma 1960s & 604 & 5 & 3.073 & 3.915 & 0.0080 & 2.923 & 3.805 & 0.0062 & A \\
\hline Oklahoma 1950s & 580 & 5 & 3.182 & 3.973 & 0.0407 & 2.555 & 3.473 & 0.0094 & A \\
\hline OK, KS, NE 1950-99 & 6755 & 5 & 2.566 & 3.509 & 0.0077 & 2.276 & 3.193 & 0.0023 & A \\
\hline United States east of CO 1990-2000 & 10375 & 5 & 1.867 & 2.386 & 0.0025 & 1.987 & 2.572 & 0.0022 & A \\
\hline Eastern United States 1950-95 & 17327 & 5 & 3.278 & 4.061 & 0.0403 & 2.646 & 3.588 & 0.0053 & A \\
\hline NY, New England 1950-99 & 688 & 4 & 3.798 & 3.850 & 0.0833 & 2.277 & 2.820 & 0.0009 & A \\
\hline Eastern CO 1950-99 & 871 & 4 & 2.738 & 2.891 & 0.0127 & 2.017 & 2.140 & 0.0007 & B \\
\hline FL 1950-95 & 2168 & 4 & 2.884 & 3.071 & 0.0070 & 2.563 & 2.803 & 0.0041 & B \\
\hline FL $1990-2000$ & 798 & 3 & 1.309 & 1.099 & 0.0001 & 1.235 & 0.971 & 0.0000 & B \\
\hline CA, OR, WA 1950-99 & 327 & 3 & 3.473 & 3.162 & 0.0001 & 3.362 & 3.106 & 0.0000 & $\mathrm{C}$ \\
\hline Front Range 1950-99 & 304 & 3 & 3.756 & 3.171 & 0.0191 & 2.123 & 2.042 & 0.0000 & $\mathrm{C}$ \\
\hline Front Range/West Coast 1950-95 & 631 & 3 & 3.595 & 3.165 & 0.0046 & 2.791 & 2.703 & 0.0000 & $\mathrm{C}$ \\
\hline Argentina $1930-1979$ & 368 & 5 & 1.719 & 2.264 & 0.0076 & 1.191 & 1.196 & 0.0008 & A \\
\hline France $1680-2000$ & 312 & 5 & 4.507 & 4.796 & 0.0485 & 4.785 & 4.875 & 0.0454 & A \\
\hline France $1680-1999$ & 294 & 5 & 4.588 & 4.839 & 0.0633 & 4.934 & 4.933 & 0.0559 & A \\
\hline Germany 855-2004 & 557 & 5 & 3.561 & 4.077 & 0.1570 & 2.174 & 2.924 & 0.0031 & A \\
\hline Germany $1453-2003$ & 417 & 5 & 3.400 & 4.017 & 0.2304 & 1.658 & 2.188 & 0.0001 & A \\
\hline Germany $1453-2002$ & 325 & 5 & 3.564 & 4.011 & 0.3101 & 1.612 & 2.010 & 0.0008 & A \\
\hline Germany 1453-2001 & 272 & 5 & 3.655 & 4.093 & 0.3381 & 1.650 & 2.120 & 0.0014 & A \\
\hline Germany $1453-2000$ & 223 & 5 & 3.799 & 4.186 & 0.3747 & 1.689 & 2.223 & 0.0003 & A \\
\hline Australia 1795-1999 & 239 & 4 & 2.751 & 3.253 & 0.0236 & 3.821 & 3.896 & 0.0000 & A \\
\hline Canada 1950-98 & 625 & 4 & 2.110 & 2.641 & 0.0091 & 2.932 & 3.433 & 0.0003 & A \\
\hline Soviet Union $1795-86$ & 221 & 4 & 3.179 & 3.547 & 0.0236 & 2.282 & 2.822 & 0.0033 & A \\
\hline South Africa 1905-2002 & 204 & 4 & 4.294 & 4.056 & 0.0261 & 3.854 & 3.902 & 0.0143 & A \\
\hline South Africa 1905-95 & 195 & 4 & 4.485 & 4.107 & 0.0426 & 3.747 & 3.844 & 0.0093 & A \\
\hline South Africa 1905-90 & 174 & 3 & 5.567 & 4.171 & 0.0787 & 3.240 & 3.607 & 0.0000 & A \\
\hline Italy $1990-99$ & 158 & 3 & 5.816 & 3.949 & 0.0611 & 3.592 & 3.422 & 0.0000 & B \\
\hline Japan 1961-2000 & 334 & 3 & 4.099 & 3.83 & 0.0003 & 4.236 & 3.871 & 0.0000 & B \\
\hline United Kingdom 1950-2002 & 1047 & 3 & 5.750 & 3.513 & 0.1397 & 2.298 & 1.960 & 0.0000 & $\mathrm{C}$ \\
\hline United Kingdom 1950-1997 & 944 & 3 & 5.968 & 3.560 & 0.1617 & 2.287 & 1.961 & 0.0000 & $\mathrm{C}$ \\
\hline
\end{tabular}

Here we use $a=-2$ and $F=n+0.5$ for each discrete F-scale class $\mathrm{F}_{n}$, and $N_{0}$ is the total number of events. From Eq. (3) we can derive the occurrence ratio

$$
\begin{aligned}
\frac{N(F)}{N(F-1)} & =\frac{\left(\frac{F+2}{b}\right)^{c-1} \exp \left[-\left(\frac{F+2}{b}\right)^{c}\right]}{\left(\frac{F+1}{b}\right)^{c-1} \exp \left[-\left(\frac{F+1}{b}\right)^{c}\right]} \\
& =\left(\frac{F+2}{F+1}\right)^{c-1} \exp \left[-\left(\frac{F+2}{b}\right)^{c}+\left(\frac{F+1}{b}\right)^{c}\right],
\end{aligned}
$$

$$
\begin{aligned}
\ln \frac{N(F)}{N(F-1)}= & (c-1) \ln \frac{F+2}{F+1}-\left(\frac{F+2}{b}\right)^{c} \\
& +\left(\frac{F+1}{b}\right)^{c} .
\end{aligned}
$$

For a given ratio, this nonlinear equation has to be solved numerically in order to derive the $b(c)$ relation. Using $R(\mathrm{~F} 4 / \mathrm{F} 3)=0.238$ from above, we obtain the solid line $b(c)$ shown in Fig. 1, which reproduces the observed correlation in the $c-b$ diagram very well. This indicates once more a similar "universal" climatology for the data samples with points close to this line. 


\section{b. Extracting a climatological Weibull distribution from U.S. decadal data}

The $c, b$ points derived from the U.S. decadal data from 1950 to 1999 as shown in Fig. 1 reveal a monotonic behavior with time. Dotzek et al. (2003) interpreted this as a convergence toward climatological parameters $c$ and $b$ of a stationary probability density distribution $p^{*}$. To assume stationarity of $p^{*}$ is also supported by the third Intergovernmental Panel on Climate Change (IPCC) Assessment Report (Houghton et al. 2001). The observed distribution can then be written as the product of $p^{*}(F)$ and a detection efficiency function $p_{\mathrm{d}}(F)$. The latter is defined as the probability to observe and classify an event as a tornado of a certain intensity $F^{3}$ Thus, based on this definition a detected, but unclassified, tornado will not occur in the observed intensity distribution. This is important because, for example, in the United States since 1982, observed tornadoes without rated damage to man-made structures have been assigned as F0 events no matter how high the wind speeds may actually have been (Brooks 2004). Under the assumption of a temporal invariance of the asymptotic distribution $p^{*}$, we can extract $p^{*}$ and the time-dependent $p_{\mathrm{d}}$ from the U.S. decadal data by a simultaneous fitting of fixed Weibull parameters and variable detection probabilities to the multiple U.S. dataset with respect to five decades starting in 1950. The fit function is defined as follows:

$$
\begin{aligned}
N(F) & =N_{0} p^{*}(F) p_{\mathrm{d}}(F), \\
p_{d}(F) & =1-d \exp \left\{-[(F-f) / w]^{2.7}\right\}(F \geq f),
\end{aligned}
$$

where $N_{0}$ is the total number of tornadoes per decade and $d, f$, and $w$ are the time-dependent fit parameters. At low $F, 1-d$ is the detection probability and $w$ is the width of the detection efficiency function. Before the fitting procedure, the decadal data have been normalized with respect to the average number of F4 observations in order to remove fluctuations in the total number of events among the different decades. For the 1980s and 1990s F0 data were excluded from the fit because they may have also contained tornadoes of a higher intensity without having rated damage. This requires also an adjustment of the shift parameter $f$, which is chosen to yield a best fit with $f=-0.5$ for the $1950 \mathrm{~s}$ to $1970 \mathrm{~s}$ and $f=1.5$ for the $1980 \mathrm{~s}$ and $1990 \mathrm{~s}$, respectively. The result of the fitting procedure is given in Table 2. The Weibull parameters $c$ and $b$ as well as the total number of events $N_{0}$ were shared by all five datasets as global fit parameters. Figure 2a shows the

\footnotetext{
${ }^{3}$ Further, one could introduce a probability distribution for the error width in assigning the appropriate $\mathrm{F}$ scale. This would then lead to a convolution instead of a simple multiplication. For the sake of simplicity, and due to the lack of information about the shape of such an error distribution, we consider the simple product only.
}

TABLE 2. Result for a multiple dataset least squares fit of a climatological Weibull distribution modified with a detection efficiency function from Eqs. (5a), (5b) to the U.S. decadal data 1950-99. Uncertainties of free-fit parameters are given in

\begin{tabular}{|c|c|c|c|c|c|c|}
\hline & & $1950 \mathrm{~s}$ & $1960 \mathrm{~s}$ & $1970 \mathrm{~s}$ & $1980 \mathrm{~s}$ & $1990 \mathrm{~s}$ \\
\hline \multirow[t]{3}{*}{$\mathrm{p}^{*}$} & $\begin{array}{l}N_{0} \\
c \\
b\end{array}$ & $\begin{array}{r}69400(26 \\
1.79 \\
2.13\end{array}$ & & & & \\
\hline & & \multicolumn{3}{|c|}{$f=-0.5$} & \multicolumn{2}{|c|}{$f=1.5$} \\
\hline & $d$ & $0.982(11)$ & $0.946(23)$ & $0.944(24)$ & $0.32(13)$ & $0.49(10)$ \\
\hline $\mathrm{p}_{\mathrm{d}}$ & $w$ & $3.46(25)$ & $2.95(25)$ & $2.61(26)$ & $1.54(36)$ & $2.34(39)$ \\
\hline
\end{tabular}
parentheses.

U.S. decadal intensity distributions and the stationary intensity distribution $N^{*}=N_{0} p^{*}$. The corresponding detection efficiency functions $p_{\mathrm{d}}(F)$ are depicted in Fig. $2 \mathrm{~b}$ in comparison to the ratio of the observed data and $p^{*}(F)$, which demonstrates the consistency of the fitting procedure. The error bars are deduced from the statistical error $N^{-1 / 2}$ of the number of observed tornadoes

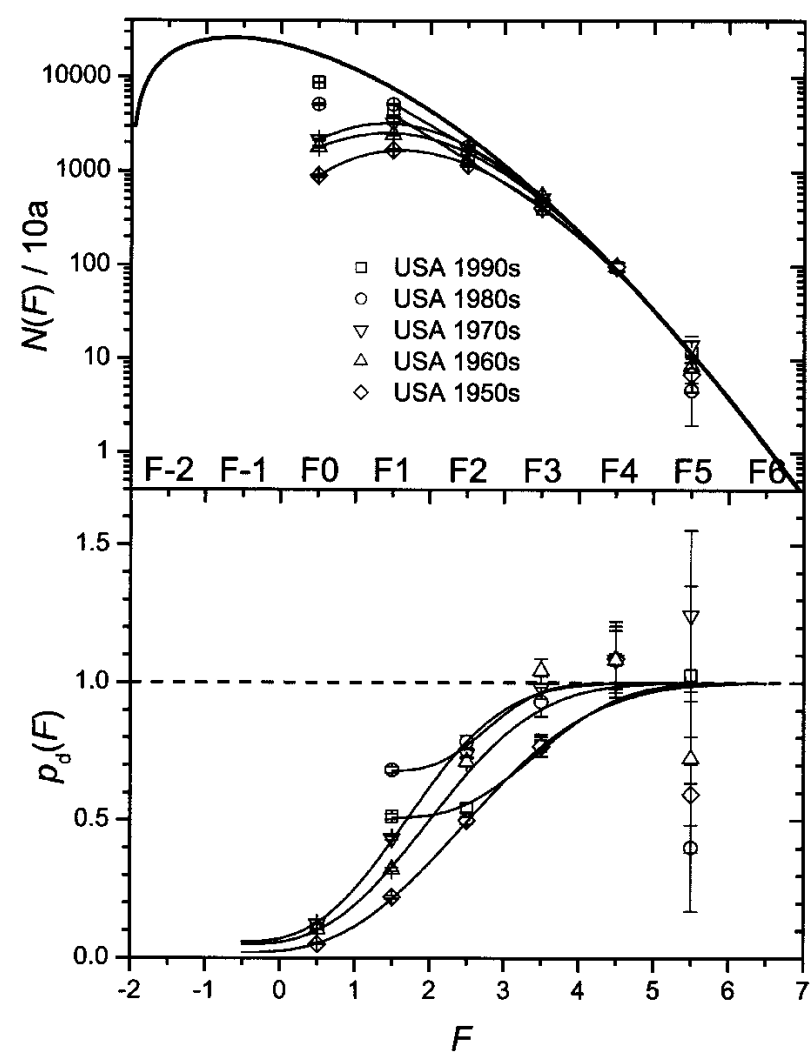

FIG. 2. (a) Multiple dataset fit to the U.S. decadal data 1950-99 using a Weibull distribution $p^{*}(F)$ multiplied with a detection efficiency function $p_{\mathrm{d}}(F)$. Thick line: climatological distribution $p^{*}(F)$; thin lines: individual distributions, including $p_{\mathrm{d}}(F)$. (b) Detection efficiency functions $p_{\mathrm{d}}(F)$ (lines) and ratio of observed ratio of the observed data and $p^{*}(F)$ (symbols) for the individual datasets. For the resulting fit parameters see Table 2 . 
for a given intensity class. The detection efficiency function gives an estimate for the degree of underreporting, which is approximately $50 \%$ for weak U.S. tornadoes $(\mathrm{F} 0, \mathrm{~F} 1)$ in the $1990 \mathrm{~s}$. As a result of the fit, we obtain from $p^{*}$ climatological Weibull parameters $c=$ $1.79(12)$ and $b=2.13(20)$ (shown as data points with error bars in Fig. 1), which can be attributed to a supercell-dominated case since presence of violent $(\mathrm{F} 4$, F5) tornadoes is apparently an indicator for supercell tornadogenesis (see sections 4 and 5 for further discussion). How well this distribution agrees with other datasets is also seen from Fig. 1, which shows the $c, b$ dependence (dashed curve) for a constant occurrence ratio $R(\mathrm{~F} 4 / \mathrm{F} 3)=0.177$ calculated from $p^{*}$.

Knowledge of the climatological Weibull parameters allows for a calculation of the relative occurrence of tornado intensities including subcritical (negative F) and superviolent events (F6), as given in Table 3. Comparing the calculated values with the U.S. data from the 1990s, we find reasonable agreement. Also, the tentative estimate of F6 tornadoes being roughly a 10-yr event in the United States (Dotzek et al. 2003) is reproduced by our present results. These data are useful for both improved tornado risk assessment and a climatological characterization of other regions worldwide (see also sections 4 and 5). The largest uncertainty of the fitting result concerns the number of subcritical tornadoes. However, this extrapolation is more academic since such weak vortices will remain very difficult to detect.

\section{The Fo problem and subcritical tornadoes}

In their previous work, Dotzek at al. (2003) used pseudolinear regression-fitting procedures of the cumulated probability from Eq. (2b) in a linearized form:

$$
\ln [-\ln (1-P(x))]=c \ln (x-a)-c \ln b,
$$

which has been found to be the most stable method. To obtain the Weibull parameters for various regions, their "Procedure II" treated the number of subcritical tornadoes (negative F) as a free-fit parameter and included the observed F0 events as a separate class. In several cases, strong underreporting of F0 events forced the fit to comparatively large $c$ values that resulted in an underestimation of the stronger tornadoes $(\mathrm{F} 4, \mathrm{~F} 5)$. Other problems can emerge from events rated as F0 not being distinguished from subcritical vortices or result from an established rating practice (e.g., apparently in the United States) if no damage to man-made structures is reported. To circumvent this, "Procedure I" from Dotzek et al. (2003) may be used. In this case the number of F0 events is treated as an unknown and the sum of subcritical and F0 tornadoes (F-2 to F0) is a free parameter. In fact, Fig. 3 reveals that Procedures I and II are special cases of our $p_{\mathrm{d}}$ concept: We have $p_{\mathrm{d}}=\theta(-1)$ for Procedure I and $p_{\mathrm{d}}=\theta(0)$ for Procedure II, respectively, where $\theta$ is the unit step function

$$
\theta(x)=\left\{\begin{array}{ll}
1 & x \geq 0 \\
0 & x<0
\end{array} .\right.
$$

In the following, the effects of these special cases of $p_{\mathrm{d}}$ functions will be demonstrated for the Center of Competence for Severe Local Storms in Germany, Austria, and Switzerland (TorDACH) version 1.4.00 (available online at http://tordach.org), as of spring 2004.

\section{a. Example: Data from Germany 855-2004}

Figure 4 shows the tornado intensity distribution for Germany based on 557 intensity-rated observations between the years 855 and 2004 (about 65\% of all reported events, most of which occurred between 1880

TABLE 3. Absolute 10-yr numbers and percentage of tornado occurrence calculated for the U.S. using the climatological Weibull parameters given in Table 1 compared to the U.S. 1990s data. The variability of calculated values emerging from the uncertainty in the

\begin{tabular}{|c|c|c|c|c|c|c|}
\hline \multirow[b]{2}{*}{ F scale } & \multicolumn{4}{|c|}{ Climatological Weibull fit in $F$} & \multicolumn{2}{|c|}{ U.S. $1990 \mathrm{~s}$} \\
\hline & $N$ & & & $\%(\mathrm{~F} 0-\mathrm{F} 6)$ & $\mathrm{N}$ & $\%$ \\
\hline $\mathrm{F}-2$ & 15 800(9917) & & 22.8 & & - & - \\
\hline $\mathrm{F}-1$ & $25197(12277)$ & 59.1 & 36.3 & & - & - \\
\hline F0 & $17448(4508)$ & & 25.1 & 61.4 & 7370 & 60.7 \\
\hline F1 & 7796(1190) & & 11.2 & 27.5 & 3274 & 27.0 \\
\hline $\mathrm{F} 2$ & $2466(213)$ & & 3.55 & 8.68 & 1065 & 8.77 \\
\hline F3 & $576.0(349)$ & & 0.83 & 2.03 & 339 & 2.79 \\
\hline F4 & $101.8(81)$ & & 0.147 & 0.358 & 81 & 0.667 \\
\hline F5 & $13.8(20)$ & & 0.02 & 0.049 & $10 / 9^{*}$ & $0.082 / 0.0074$ \\
\hline F6 & $1.4(4)$ & 40.9 & 0.002 & 0.005 & $0 / 1^{*}$ & $0.0 / 0.0008$ \\
\hline$\Sigma \mathrm{F} 0-\mathrm{F} 6$ & $28403(5956)$ & & & & 12139 & \\
\hline$N_{0}$ & $69400(26$ 150) & & & & - & \\
\hline
\end{tabular}
parameters is given in parentheses.

* In the official U.S. tornado database, the Bridge Creek Tornado (OK, 3 May 1999) is rated as F5. Yet, Doppler measured near-surface wind speeds (or rather debris particle velocities) at the F5-F6 threshold. An F6 classification of this event would still match the extrapolated probability based on the climatological Weibull fit. 


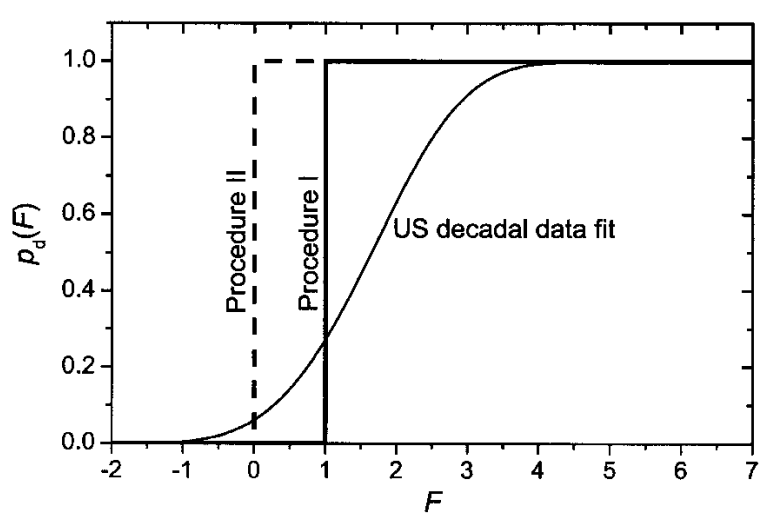

FIG. 3. Detection efficiency functions $p_{\mathrm{d}}(F)$ (schematic) for fitting Procedures I and II (step functions) and derived from the multiple U.S. decadal dataset fit (continuous function).

and 2004) in comparison with the Weibull fits using Procedures I (solid curve) and II (dashed curve). Procedure II results in a best fit with $c=3.592, b=4.089$, and $N_{0}=599$, that is, $N(\mathrm{~F}-2$ to $\mathrm{F}-1)=45$. The result of Procedure I, however, is $c=2.174, b=2.924$, and $N_{0}$ $=1338$, that is, $N(\mathrm{~F}-2$ to $\mathrm{F} 0)=877$. In the latter case, exclusion of the F0 tornado reports in the fitting procedure brings the Weibull distribution in much better agreement with the observed F1-F5 data and the $c, b$ parameters close to the climatological values obtained from the U.S. decadal data. The fit is also selfconsistent, as the "input" of $N_{\text {in }}(\mathrm{F}-2$ to F1) $=1140$ coincides very well with the corresponding value calculated from the cumulative Weibull distribution $N_{\text {out }}(\mathrm{F}-2$ to $\mathrm{F} 1)=1152$.

\section{b. Worldwide results excluding FO observations}

The result of fitting Procedure I for various regions worldwide with sufficiently large databases (more than

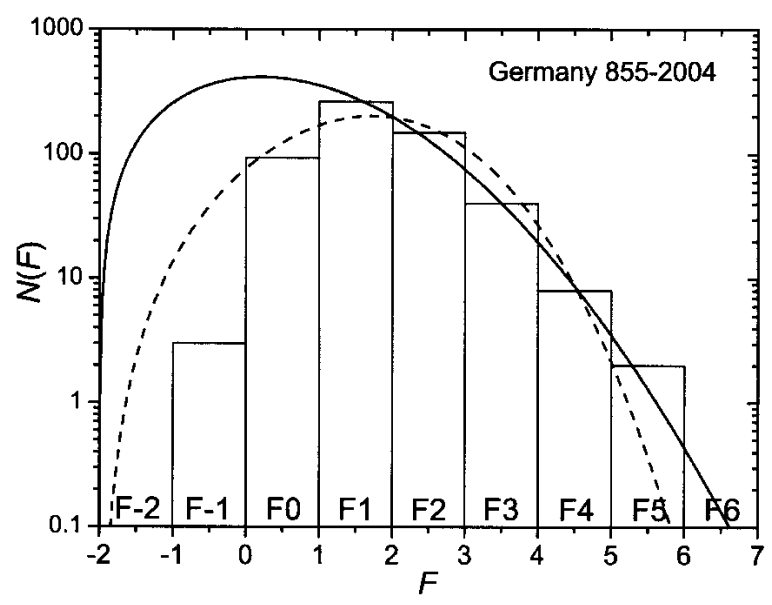

FIG. 4. German tornado intensity data from 855 to 2004 . Solid line: Weibull fit in $F$ excluding F0 data; dashed line: Weibull fit in $F$, including F0 data. See also the text and Table 1.
100 events above F0 intensity) is given in Table 1 in comparison with the values for $c$ and $b$ from Dotzek et al. (2003), using Procedure II. As shown in Fig. 5, most of the data for Procedure I (bullets) in the $c-b$ diagram are shifted toward lower $c$ values, indicating a better convergence compared to Procedure II (circles). The self-consistency of the fit can either be measured by the ratio of the input number of weak/subcritical tornadoes and the corresponding output (modeled) number from the cumulative distribution as a consistency parameter,

$$
\begin{aligned}
r_{c}= & N_{\text {in }}(\mathrm{F}-2 \text { to } \mathrm{F} 1) / N_{\text {out }}(\mathrm{F}-2 \text { to } \mathrm{F} 1), \\
& \text { F0 reports excluded, Procedure I } \\
r_{\mathrm{c}}= & N_{\text {in }}(\mathrm{F}-2 \text { to } \mathrm{F} 0) / N_{\text {out }}(\mathrm{F}-2 \text { to } \mathrm{F} 0),
\end{aligned}
$$$$
\text { F0 reports included, Procedure II }
$$

or by the well-known $\chi^{2}$ parameter (e.g., Press et al. 1992). Treating the F0 reports as an unknown in the Weibull fit yields a convincing improvement of both the self-consistency $r_{c}$ (see inset in Fig. 5) and the $\chi^{2}$ values (see Table 1), demonstrating that Procedure I is superior to Procedure II in this respect.

A few data points (8 out of 44) show an increase in $c$ of more than $10 \%$ if F0 data are excluded from the fit. The corresponding databases are either quite small (e.g., Australia and Canada) or show a large number of F0 events $[N(\mathrm{~F} 0)>N(\mathrm{~F} 1)$, e.g., Oklahoma in the 1990s, supposedly caused by subcritical tornadoes or tornadoes without rated damage being assigned as F0] or a strong F2 underreporting $[N(\mathrm{~F} 2)<N(\mathrm{~F} 3)$, e.g., France]. However, even after a generally improved convergence, we still find a significant number of data points in the $c-b$ diagram below the curve of constant $R(\mathrm{~F} 4 / \mathrm{F} 3)=0.177$. Thus, the question arises whether a different climatology is associated with these data points. This is addressed in the following section.

\section{Worldwide tornado intensity distributions and corresponding climatology classes}

\section{a. $N(F) / N(F-1)$ ratios for various regions worldwide}

As shown in section 2, the occurrence ratio $R(\mathrm{~F} 4 / \mathrm{F} 3)$ seems to reflect a climatological invariant, which agrees well with the data points in the $c-b$ diagram for regions with observed F5 tornadoes. This ratio corresponds to a part of the average slope of the intensity distribution, which has been hypothesized as an indicator for the nature of tornadogenesis processes (Brooks and Doswell 2001b; Dotzek et al. 2003). In the following, we will extend this analysis from the average slope to the individual $N(F) / N(F-1)$ ratios $(F=2,3,4)$ with respect to those datasets without F5 or F4 events, respectively. Figure 6 shows the ratios for the data records from various regions worldwide. All datasets including 


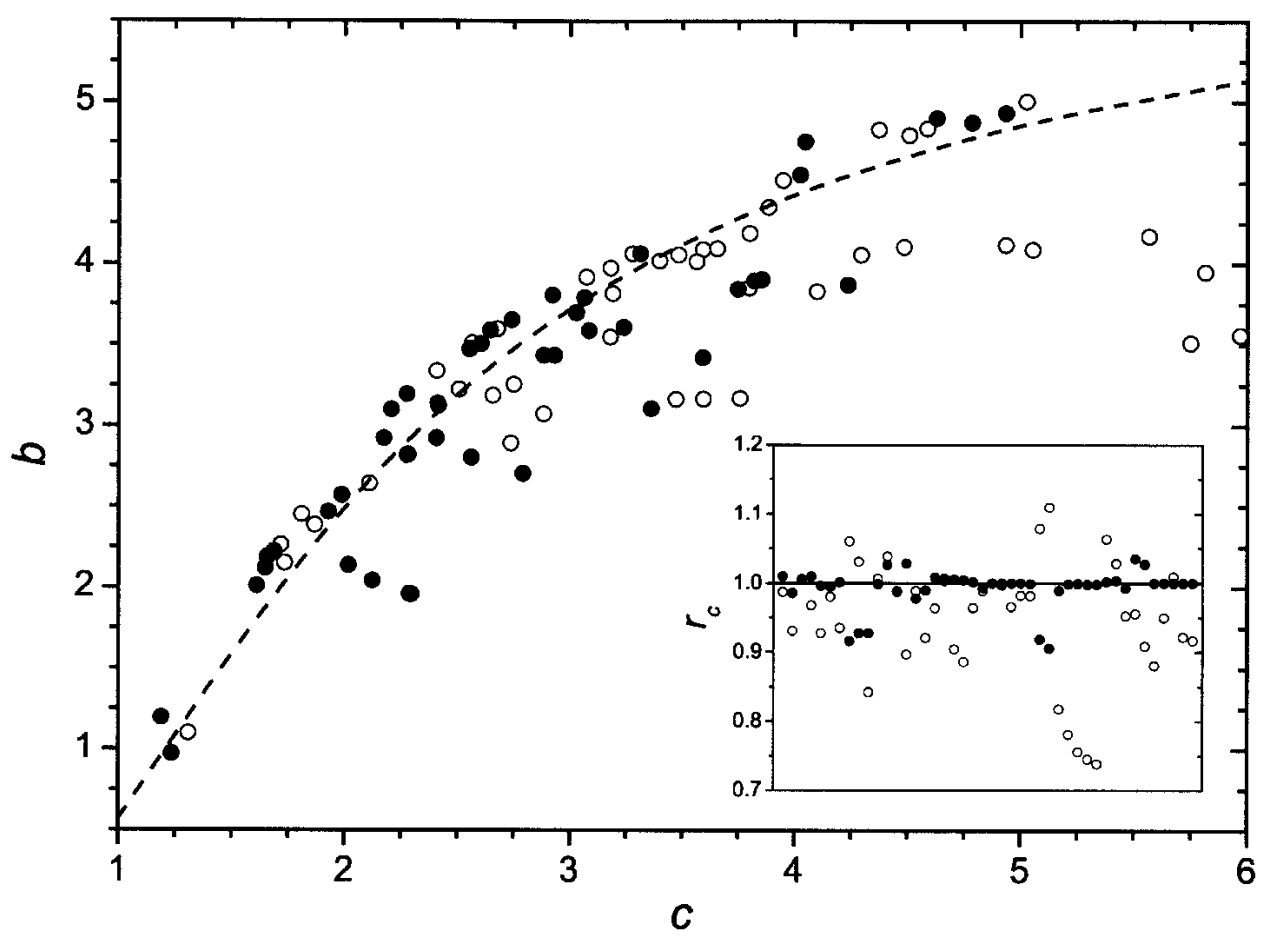

FIG. 5. The $c-b$ diagram of Weibull parameters $c$ and $b$; $:$ results from Weibull fit in $F$ excluding F0 data (Procedure I); O: results from Weibull fit in $F$ including F0 data (Procedure II, from Dotzek et al. 2003). Dashed line: curve of constant occurrence ratio $R(\mathrm{~F} 4 / \mathrm{F} 3)=0.177$ (from U.S. decadal fit). The inset shows values of the consistency parameter $r_{\mathrm{c}}$ as defined in Eqs. (7a), (7b). See also text and Table 1.

observed F4 events show a comparable $N(4) / N(3)$ ratio. Considering the $N(3) / N(2)$ ratio, we get again similar values among the F4-including sets except for the data from eastern Colorado and Florida. The latter show significantly lower ratios as for Italy and Japan. The lowest $N(3) / N(2)$ ratios are found for the U.S. West Coast and the Front Range and for the United Kingdom. Based on this analysis, we can distinguish three groups. Group A comprises the regions with F4including tornado records except for eastern Colorado and Florida. The corresponding average ratios are $R_{\mathrm{A}}(\mathrm{F} 4 / \mathrm{F} 3)=0.230(12),{ }^{4} R_{\mathrm{A}}(\mathrm{F} 3 / \mathrm{F} 2)=0.318(11)$, and $R_{\mathrm{A}}(\mathrm{F} 2 / \mathrm{F} 1)=0.468(24) .{ }^{5}$ For group C, we find values of $R_{\mathrm{C}}(\mathrm{F} 2 / \mathrm{F} 1)=0.129(34)$ and $R_{\mathrm{C}}(\mathrm{F} 3 / \mathrm{F} 2)=0.048(11)$. Aside from the absence of $\mathrm{F} 4$ events, this indicates a difference in the climatology compared to group A. Group B likely is an intermediate case with a possibly bimodal $p^{*}(F)$. A tentative assignment for the climatology of the various regions being of type $\mathrm{A}, \mathrm{B}$, or $\mathrm{C}$ is given in Table 1.

\footnotetext{
${ }^{4}$ Compare to $R(\mathrm{~F} 4 / \mathrm{F} 3)=0.238(13)$ from the F5-including subset of group A (section 2).

${ }^{5}$ This is excluding the U.S. decadal data before 1950 and the French data because they show a strong underreporting for weak tornadoes.
}

\section{b. The $c-b$ diagram as a chart for tornado climatology}

To discuss these indications in more detail, we will consider the behavior of the various regions and apparent invariants in the $c-b$ diagram. Figure 7 shows the $b(c)$ curves of constant $N(F) / N(F-1)$ ratios corresponding to groups $\mathrm{A}$ and $\mathrm{C}$ and $c, b$ data pairs from selected regions. For each of these regions, the fitting results with F0 data included and excluded are given (cf. Table 1) in order to demonstrate their convergence behavior. The shaded areas cover those parts of the $c-b$ diagram associated with invariant $N(F) / N(F-1)$ ratios of group A (upper area; $F=2,3,4$ ) and group $\mathrm{C}$ (lower area; $F=2,3)$. For large values $(c>4)$, these areas start to overlap, whereas at lower $c$ values we find two well-separated bands. All data points of groups A and $\mathrm{C}$, respectively, fit nicely to the corresponding areas in the $c-b$ diagram and are also clearly separated by their convergence behavior (exclusion of F0 in the Weibull fit). This consistency again gives a strong indication, if not even evidence, for the existence of at least two different types of tornado climatology, and proves the $c-b$ diagram to be a useful climatological chart. In section 5 we will give an interpretation within the context of the previously discussed relation of the average slope of intensity distributions to the physical processes for tornadogenesis. 


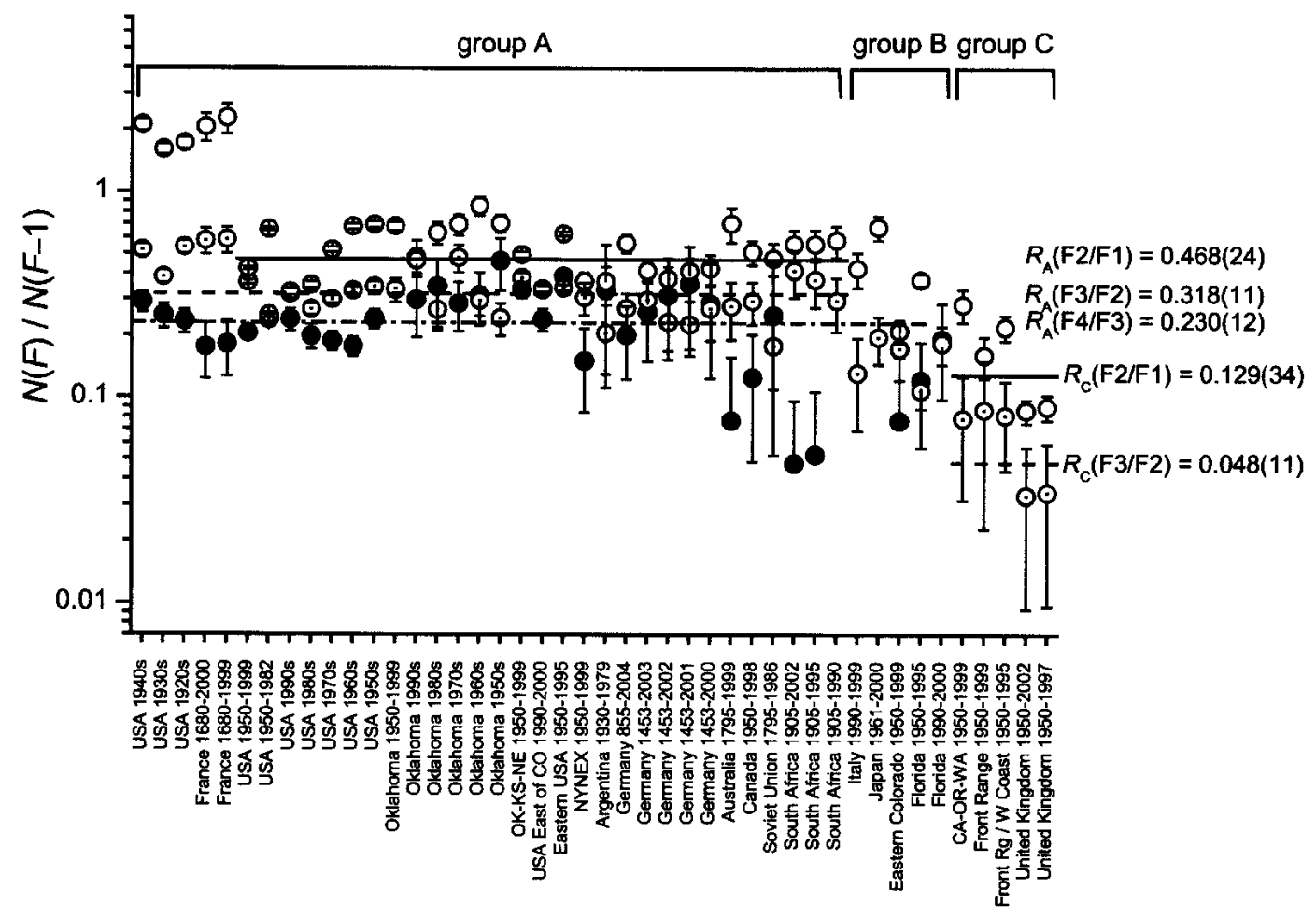

FIG. 6. Occurrence ratios $N(F) / N(F-1)(F=2,3$, 4) for various regions worldwide. Groups with similar ratios: A (presumably supercell dominated), C (presumably nonsupercell dominated), B (bimodal case). Group-specific mean ratios are shown as horizontal lines: solid, $R(\mathrm{~F} 2 / \mathrm{F} 1)$; dashed, $R(\mathrm{~F} 3 / \mathrm{F} 2)$; chain, $R(\mathrm{~F} 4 / \mathrm{F} 3)$.

\section{Discussion}

In the following, we consider our present assessment of tornado climatology through global tornado intensity distributions in the context of previous studies. Brooks and Doswell (2001b) presented a first comparative study of intensity distributions from various regions worldwide. Their analysis revealed an exponential-like behavior of the distributions being characterized by a nearly uniform slope in a lin-log plot. Since exponentials are determined by a single parameter, exponentials can be considered as a "first order" approach. Two distinct distributions-one apparently associated with supercell tornadogenesis processes and the other with nonsupercell processes-were found in both the U.S. data and in other countries. The lack of a physical boundary condition (zero probability at zero wind speed) for exponentials, and the curvature to the right of observed intensity distributions at larger $F$ in lin-log plots due to the inherent upper limit of tornado intensity near the F5-F6 threshold, led to the use of Weibull distributions (Dotzek et al. 2003). A further improvement is the introduction of a detection efficiency function $p_{\mathrm{d}}(F)$. The latter was-in the form of two special cases-already included in the fitting Procedures I and II from Dotzek et al. (2003). Here, $p_{\mathrm{d}}(F)$ is approximated by a unit step function at $F=1$ (Procedure I) or at $F=0$ (Procedure II), respectively (see Fig. 3). In both cases, we have no (Procedure II) or at least no detailed (Procedure I) information on $p_{\mathrm{d}}(F)$. Whereas Procedure II requires efficient detection of F0 tornadoes, Procedure I gives quite good fitting results, even for tornado records with strong F0 underreporting (see section 3). The effect of $p_{\mathrm{d}}(F)$ on the observed distributions was qualitatively discussed by Dotzek et al. (2003) (cf. their Fig. 7). In the present work a continuous detection efficiency function $p_{\mathrm{d}}(F)$ is explicitly introduced for the "global" fit to the U.S. decadal data (1950-99). Although this procedure works only for such large databases, it yields information about the likely stationary intensity distribution $p^{*}(F)$ in the form of the parameters $N_{0}, c$, and $b$. The Weibull parameters $c$ and $b$ can be applied to other regions worldwide with similar intensity distributions and can help to estimate the detection efficiency $p_{\mathrm{d}}(F)$ there. Further, knowledge of $p^{*}(F)$ is very useful for risk assessment and analysis, for example, to estimate the probability of violent tornado occurrences (including hypothetical F6 tornadoes) and recurrence intervals.

The average slope between F2 and F4 in lin-log intensity distributions used by Brooks and Doswell (2001b) for climatological characterization is related to the $c-b$ correlation discussed in our present work. By extension of the average slope (exponential) to individual ratios $R\left(\mathrm{~F}_{n} / \mathrm{F}_{n-1}\right)$ of neighboring F-class occurrence (Weibull), we are able to associate distinct areas 


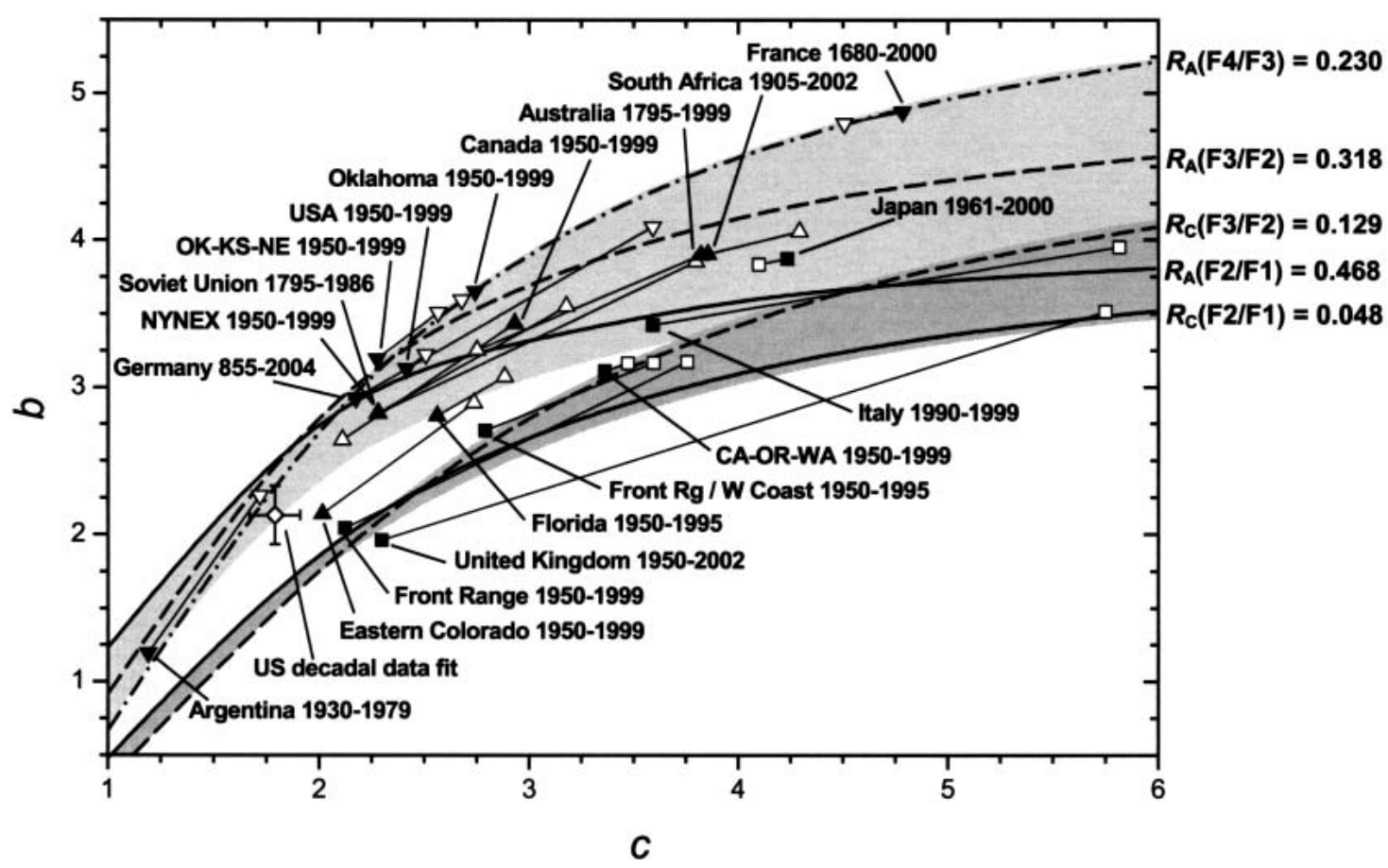

FIG. 7. A $c-b$ diagram of Weibull parameters $c$ and $b$ as a climatological chart. Areas (shaded) of invariant ratios $R=N(F) / N(F-1)$ for group A (upper area, $F=2,3,4$ ) and group $\mathrm{C}$ (lower area, $F=2,3$ ) and corresponding curves of constant $R$ (see also Fig. 6); $c, b$ data for selected regions, showing their convergence behavior under exclusion of F0 observations from the fit: full symbols, Procedure I (F0 data excluded); open symbols, Procedure II (F0 data included).

in the $c-b$ diagram to different groups $(\mathrm{A}, \mathrm{B}, \mathrm{C}$; see Fig. 6 and section 4). Following Brooks and Doswell (2001b) these differences may be a result of the physical processes leading to tornadogenesis in those regimes. Regions belonging to group $\mathrm{A}$, such as, for example, the so-called tornado alley (Oklahoma, Kansas, Nebraska), appear to be dominated by supercell tornado events. Whereas the occurrence of violent (F4, F5) events is an indicator for supercell tornadogenesis, the situation for group C is less evident. Here, we find regions like the U.S. Front Range and West Coast, or the United Kingdom. The Front Range of Colorado has many so-called landspouts (e.g., Brady and Szoke 1989), which appear to hardly exceed F2 intensity. However, from several case studies, we can merely hypothesize that those regions with similar intensity distributions show, in fact, a dominance of nonsupercell tornadogenesis. A few regions (group B) are found in an intermediate situation regarding their location in the $c-b$ diagram and their convergence behavior. Florida and eastern Colorado seem to be closer to group $\mathrm{C}$ but show some F4 events from a presumed supercell "background." Japan seems to be close to group A (similar to South Africa), although no violent tornadoes have been reported, probably due to the relatively short sampling time. The latter also concerns Italy, in particular, where supercell tornadoes are well known, but violent events are not contained in the short-range database (1990s). Here, an acquisition of historical data is required, as was done, for example, for Germany and France. In general, more systematic analysis is needed in order to prove whether our hypothesis is valid. In this respect our study on intensity distributions and the use of the $c-b$ plot for climatological characterization is only a first step. Future work should concern the crossrelation of tornado intensity and tornadogenesis processes.

We have based the Weibull fits exclusively on the $\mathrm{F}$ scale as it has gained the most widespread acceptance worldwide. However, an adaption of our procedure to derive the $c, b$ values from tornado reports based on the $\mathrm{T}$ scale is straightforward. We have also successfully applied the Weibull fits to downburst reports as a function of the F scale. Yet, as downburst intensity is limited to F3 at most, the fits are then based on four F-scale classes only. So, especially for downburst reports but also for tornadoes, the doubled number of T-scale intensity classes compared to the $\mathrm{F}$ scale appears attractive in principle from a statistical climatological point of view. Unfortunately, quite large databases are required to fill the larger number of T-scale classes with statistically representative data samples, and reports based on the $\mathrm{T}$ scale are only available from relatively few countries in the world and often do not show adequate sample sizes. Currently, performing the Weibull fits with T-scale reports cannot provide the global perspective offered by the much more widely used F scale.

The present analysis is still based on a "practical" 
(damage related) measure of intensity. From the climatological viewpoint, a velocity-based analysis would be more appropriate. In fact, the original definition of the $\mathrm{F}$ scale is related physically to the wind speed and not related phenomenologically to the observed damage; that is, we have the defining relation $v(\mathrm{~F})$ given by Eq. (1). The damage "D" follows then from a much more complex relation $\mathrm{D}[v(\mathrm{~F})]$, which is strongly variable for different regions. Thus, the simple inversion, which treats $v(\mathrm{~F})$ as a function of a damage-related $\mathrm{F}$ scale for a specific region (e.g. the United States) will not provide a sensible solution to this dilemma (cf. Doswell and Burgess 1988; Brooks and Doswell 2001b). A much better way, which at the same time keeps the physical foundation of the ( $v$ related) scale, is the use of regionalized damage descriptions in the form of a damagerelated f scale, as suggested by Fujita (1992). The conversion of the phenomenological $\mathrm{f}$ scale into the physical F scale (which should be exclusively used for climatological considerations) is achieved by means of a regional "f-scale matrix" individually defined for each specific region worldwide. This, of course, demands a more profound knowledge of the wind speed/damage relation, that is, to find typical, observable damage. For buildings in Central Europe, Dotzek et al. (2000) introduced typical loss ratios (defined for any damaged object as occurred loss divided by reinstatement value) as a measure of observed damage related to the $\mathrm{T}$ and $\mathrm{F}$ scales (cf. Table 1 from Dotzek et al. 2003). Further information can be obtained from an analysis of wind damage to trees, presumably showing less variation all over the world. Recent studies on this topic demonstrated that at least up to T5 (i.e., high F2) intensity it is possible to distinguish even between separate T-scale values (Hubrig 2002; Hubrig 2004).

However, even with these recent improvements of the tornado intensity rating procedures, the ratings still remain a subjective judgment based on the amount of available information. The maximum intensity of a tornado might have occurred in a place where no adequate structures for reliable damage estimation have been present, for example, over open rural terrain. Or a slow-moving and relatively weak tornado may lead to high damage comparable to a stronger tornado moving at a more typical translational speed, simply due to its longer local impact time. Another bias with damagebased intensity ratings can arise if the rating is mainly done by one or only very few individuals in a given country. Here, a systematic under- or overrating might result, which can only be avoided if the responsibility for the ratings relies on a larger group of trained experts. While we are aware of these shortcomings of the present tornado intensity rating procedure worldwide, we have nevertheless shown evidence for the usefulness of the Weibull fitting of global tornado intensity distributions: its climatological signal is physically consistent and contributes to our understanding of tornadoes worldwide.

\section{Conclusions}

Extending recent work on statistical modeling of tornado intensity distributions by Dotzek et al. (2003), we analyzed the outcome of Weibull fits to the observed data from various regions worldwide with respect to climatologically relevant properties. The present study has revealed the following:

- A correlation of the Weibull parameters $c$ and $b$ found, in particular, for databases including F5 tornadoes indicates the presence of a globally invariant property of these distributions. It can be attributed to a fixed ratio of the numbers of tornadoes for neighboring $\mathrm{F}$ classes $R=N(F) / N(F-1)$. The observed correlation in the $c-b$ diagram is well reproduced by a ratio $R(\mathrm{~F} 4 / \mathrm{F} 3)=0.238$ extracted from databases with F5 observations.

- Large tornado records like the U.S. decadal data 1950-99 show a convergence with time along this $b(c)$ curve. Assuming temporal invariance of the climatology and using a detection efficiency function $p_{\mathrm{d}}(F)$ for tornado observations, we extracted a stationary probability distribution $p^{*}(F)$ from the U.S. decadal data with Weibull parameters $c=1.79(12)$ and $b=2.13(20)$. These can be used for risk assessment and comparative studies on tornado intensity distributions worldwide.

- The detection efficiency function $p_{\mathrm{d}}(F)$ also gives an estimate for the degree of underreporting, which appears to be approximately $50 \%$ for weak U.S. tornadoes (F0, F1) in the 1990s.

- The number of observed F0 tornadoes can give significant uncertainty to a Weibull fit due to likely inherent strong underreporting, difficult segregation from subcritical vortices, and current rating practices (e.g., the United States). We demonstrated that excluding F0 report data from our analysis improves the quality of the Weibull fits significantly.

- Extending previous considerations on the average slope of intensity distributions, based on the individual $N(F) / N(F-1)$ ratios $(F=2,3,4)$, at least two types of intensity distributions (and bimodal cases) can be distinguished, which may be related to a dominance of supercell and nonsupercell tornadogenesis, respectively. In the $c-b$ diagram, constant $N(F) /$ $N(F-1)$ ratios define separate areas that are consistent with the Weibull parameters extracted from observations. Thus, the $c-b$ diagram represents a useful chart for worldwide tornado climatology and characterization of intensity distributions.

Future work should focus on an improvement of the databases, for example, a review of historical events and enhancement of probabilities of detection for the weaker cases. For the latter also more systematic investigation on their genesis is desirable. Here, the regions from group $\mathrm{C}$ are of particular interest. If it can be proven that nonsupercell tornadogenesis is, in general, 
responsible for this type and provided that a sufficiently large database will be available allowing for detailed analysis like for the U.S. decadal data, the stationary intensity distribution for nonsupercell tornadoes could be extracted. This would be very helpful for the analysis of intensity distributions from regions that may significantly experience both types of tornadoes, that is, have a bimodal $p^{*}(F)$.

Acknowledgments. The authors would like to thank those individuals and organizations who provided the global tornado intensity data used in our study, especially Harold Brooks of NOAA/NSSL as well as the TorDACH (http://tordach.org) and ESSL (http:// essl.org) networks.

\section{REFERENCES}

Brady, R. H., and E. J. Szoke, 1989: A case study of nonmesocyclone tornado development in northeast Colorado: Similarities to waterspout formation. Mon. Wea. Rev., 117, 843-856.

Brooks, H. E., 2000: The climatology of severe thunderstorms: What we can know. Preprints, 20th Conf. on Severe Local Storms, Orlando, FL, Amer. Meteor. Soc., 126-129.

_ 2004: On the relationship of tornado path length and width to intensity. Wea. Forecasting, 19, 310-319.

— tornado climatology. Mon. Wea. Rev., 116, 495-501.

—_, and C. A. Doswell III, 2001a: Normalized damage from major tornadoes in the United States: 1890-1999. Wea. Forecasting, 16, 168-176.

- , and $-2001 \mathrm{~b}$ : Some aspects of the international climatology of tornadoes by damage classification. Atmos. Res., 56, 191-202.

Dotzek, N., 2001: Tornadoes in Germany. Atmos. Res., 56, $233-$ 251. [Available online at http://www.tordach.org/.]

- 2002: Severe local storms and the insurance industry. $J$. Meteor., 26, 3-12. [Available online at http://www.tordach. org/.]

- G. Berz, E. Rauch, and R. E. Peterson, 2000: Die Bedeutung von Johannes P. Letzmanns "Richtlinien zur Erforschung von Tromben, Tornados, Wasserhosen und Kleintromben" für die heutige Tornadoforschung (The relevance of Johannes P. Letzmann's "Guidelines for research on tornadoes, waterspouts, and whirlwinds" for contemporary tornado research). Meteor. Z., 9, 165-174. [Available online at http:// www.tordach.org/.]
_ J. Grieser, and H. E. Brooks, 2003: Statistical modeling of tornado intensity distributions. Atmos. Res., 67-68, 163-187. [Available online at http://www.tordach.org/.]

Fujita, T. T., 1981: Tornadoes and downbursts in the context of generalized planetary scales. J. Atmos. Sci., 38, 1511-1534.

_ 1992: Mystery of Severe Storms. Chicago University Press, $298 \mathrm{pp}$.

_ and A. D. Pearson, 1973: Results of FPP classification of 1971 and 1972 tornadoes. Preprints, Eighth Conf. on Severe Local Storms, Denver, CO, Amer. Meteor. Soc., 142-145.

Goliger, A. M., R. V. Milford, B. F. Adam, and M. Edwards, 1997: Inkanyamba-Tornadoes in South Africa. United Litho, 77 pp.

Houghton, J. T., Y. Ding, D. J. Griggs, M. Noguer, P. J. van der Linden, X. Dai, K. Maskell, and C. A. Johnson, Eds., 2001: Climate Change 2001: The Scientific Basis. Cambridge University Press, $881 \mathrm{pp}$.

Hubrig, M., 2002: Tornado- und Downburst-Schadenskala für Holzgewächse basierend auf der Skalierung nach TORRO angepasst für Mitteleuropa (TorDACH) (Tornado and downburst damage scale description for woody plants based on the TORRO scale and adapted for Central Europe, TorDACH). Proc. Zweites Forum Katastrophenvorsorge, Extreme Naturereignisse-Folgen, Vorsorge, Werkzeuge, Leipzig, Germany, Deutsches Komitee für Katastrophenvorsorge, 179-186. [Available online at http://www.tordach.org/.]

_ 2004: Analyse von Tornado- und Downburst-Windschäden an Bäumen (Analysis of tornado and downburst wind damage to trees). Forst $\mathrm{Holz}$, 59, 78-84. [Available online at http://www.tordach.org/.]

Kelly, D. L., J. T. Schaefer, R. P. McNulty, C. A. Doswell III, and R. F. Abbey Jr., 1978: An augmented tornado climatology. Mon. Wea. Rev., 106, 1172-1183.

McDonald, J. R., 2002: Development of an enhanced Fujita scale for estimating tornado intensity. Preprints, 21st Conf. on Severe Local Storms, Austin, TX, Amer. Meteor. Soc., 174-177.

Meaden, G. T., 1976: Tornadoes in Britain: Their intensities and distribution in space and time. J. Meteor., 1, 242-251.

Niino, H., T. Fujitani, and N. Watanabe, 1997: A statistical study of tornadoes and waterspouts in Japan from 1961 to 1993. J. Climate, 10, 1730-1752.

Peterson, R. E., 2000: Tornadoes of the former Soviet Union. Preprints, 20th Conf. on Severe Local Storms, Orlando, FL, Amer. Meteor. Soc., 138-141.

Press, W. H., B. P. Flannery, S. A. Teukolsky, and W. T. Vetterling, 1992: Numerical Recipes: The Art of Scientific Computing. 2d ed. Cambridge University Press, 973 pp.

Teittinen, J. J., 2000: Tornadoes in Finland during the years 1997-1999. Preprints, 20th Conf. on Severe Local Storms, Orlando, FL, Amer. Meteor. Soc., 95-96. 